\title{
Solitons and Nondissipative Diffusion
}

\author{
N. Theodorakopoulos ${ }^{1,2}$ and M. Peyrard ${ }^{2}$ \\ ${ }^{1}$ Theoretical and Physical Chemistry Institute, National Hellenic Research Foundation, \\ Vasileos Constantinou 48, 11635 Athens, Greece \\ ${ }^{2}$ Laboratoire de Physique, Ecole Normale Superieure de Lyon, 46 allee d'Italie, 69007 Lyon, France
}

(Received 17 February 1999; revised manuscript received 29 June 1999)

\begin{abstract}
The dynamical correlation spectra of the Toda chain are obtained by a molecular dynamics simulation and analyzed in terms of Bethe-ansatz-based soliton phenomenology. The spectrum of local temperature fluctuations exhibits a feature which can be unambiguously assigned to solitons. Detailed linewidth analysis demonstrates the phenomenon of nondissipative soliton diffusion, i.e., the stochastic sequence of spatial shifts experienced by the soliton as it moves through the lattice and interacts with other excitations without momentum exchange. Our results provide strong support for the dynamical predictions of the finite-temperature Bethe ansatz excitation spectrum.
\end{abstract}

PACS numbers: 05.45.Yv, 05.40.-a, 05.60.Cd, 63.20.Ry

Diffusion is in general accompanied by dissipation. If a test particle is injected in a diffusing medium with a velocity above the thermal velocity, it slows down at a rate predicted by the fluctuation-dissipation theorem. This happens because the constant exchange of momentum between the test particle and the medium is equivalent to a white spectrum of random forces acting on the test particle. Weaker randomness, i.e., richer-than-white force spectra, can lead to an enhanced, nondissipative diffusion, which is not mirrored in macroscopic friction; this has been widely recognized in the context of thermal ratchets. The present paper deals with another, perhaps more extreme, example of weak randomness: the nondissipative diffusive motion of thermal solitons.

Solitons, or solitonlike objects, are common in low dimensional systems and account for a variety of phenomena where nonlinear wave propagation is especially robust (hydrodynamics, optical fibers, Josephson junctions arrays, biomolecules). In its purest form, a soliton maintains both shape and velocity as it moves through a medium; it can do that because its interaction with the other components of the medium consists of spatial shifts, "jumps," rather than momentum exchanges. Put more formally, the system is completely integrable and soliton canonical momenta are conserved action variables. At finite temperatures the sequence of spatial shifts becomes intrinsically stochastic, reflecting the randomness of the initial conditions. In the absence of any further perturbations, a soliton is thus expected to perform a stochastic motion, a random walk, in addition to its ballistic motion. As long as the system is unperturbed by an extrinsic sources (e.g., impurities), there is no macroscopic friction. Stochasticity is confined to the "random" subset of the motion, and the ensuing diffusion is thus nondissipative.

Although the above scenario for stochastic soliton motion had been proposed long ago [1-3], the corresponding "signature" in the spectrum of dynamical correlations has never been observed, either experimentally or in a numerical simulation. In fact, most of the debate has concentrated on how "conventional" diffusive processes, due to "real" perturbations from exact integrability, could effectively mask any nondissipative diffusion (e.g., [4,5]). This is indeed the typical situation if a soliton is exact only in the continuum limit; since all experiments-physical or numerical-involving thermal solitons are performed on a lattice, discreteness perturbs the exact soliton property and eventually leads to macroscopic friction and concomitant conventional diffusion.

In this paper, we report our findings, numerical and theoretical, on the finite-temperature dynamics of the Toda chain. We have chosen this particular model because it exhibits the exact soliton property on the lattice; i.e., the discreteness is a condition for integrability rather than a perturbing factor.

The classical Toda chain supports supersonic localized excitations (solitons) as well as extended, wavelike, subsonic objects [6]. The statistical distribution of the two types of objects has been determined both numerically [7] and theoretically [8]. The fundamental (zero temperature) dynamics is governed by the inverse scattering theory [9]; the thermodynamics is described by the classical limit of the Bethe ansatz (BA) [10-12]; there is as yet no consensus regarding the dynamical significance of quasienergies derived within the BA formalism [13,14]. An analysis of dynamical correlation spectra in terms of soliton phenomenology [15] (i.e., by exploiting the availability of exact equilibrium statistical mechanical properties in phonon and soliton "sectors" of phase space, in order to formulate an approximate theory of dynamical correlations) yields two essential results: (i) it shows that nondissipative (soliton and phonon) diffusion exists and is observable in a nonlinear lattice, and (ii) it allows us to identify unambiguously a soliton peak in the kinetic energy autocorrelation spectra; this assignment allows us in turn to give a precise dynamical meaning to the equilibrium BA solution 
(in particular, the soliton dispersion relation derived within the BA).

Numerical simulations. - Dynamical correlations have been observed by molecular dynamics (MD) simulations on a Toda chain of $N=1024$ atoms using a fourth-order Runge-Kutta integrator. The interaction potential was $V\left(r_{n}\right)=\exp \left(-r_{n}\right)+r_{n}-1$, where $r_{n} \equiv y_{n}-y_{n-1}$ is the difference in displacement of neighboring atoms. Initial conditions were "canonical," in the sense that (i) the velocities $\dot{y}_{n}$ were Gaussian distributed, and (ii) relative displacements $r_{n}$ were random variables distributed according to the distribution function $\exp [-\beta V(r)]$, where $\beta$ is the inverse temperature.

We have computed the spectra

$$
S_{A A}(q, \omega)=\int_{-\infty}^{+\infty} d t e^{-i \omega t} \sum_{m, n} e^{i q a(n-m)}\left\langle A_{n}(t) A_{m}(0)\right\rangle,
$$

where $A_{n}=r_{n}$ (relative displacement autocorrelations) or $A_{n}=T_{n}=\dot{y}_{n}^{2} / 2$ (kinetic energy autocorrelations); $a$ is the lattice constant, which is taken equal to unity at zero temperature. The angular brackets denote an ensemble average; ergodicity is not assumed. Typically, we implemented this by repeating the simulation for a set of 636 initial conditions. Each initial condition was integrated for 512 time units. The integration time step and the fast-Fourier transform sampling step were equal to 0.01 and 0.5 , respectively (in dimensionless time units). Figures 1 and 2 display the $r r$ and $T T$ spectra, respectively, for a temperature $T=0.25$. Results are in broad agreement with previous work [16]. The interpretation is new and reflects, among other things, the detailed knowledge achieved by the BA solution.

Phonon phase diffusion and soliton diffusion.-The numerical observations can be understood theoretically by investigating the interactions between the two types

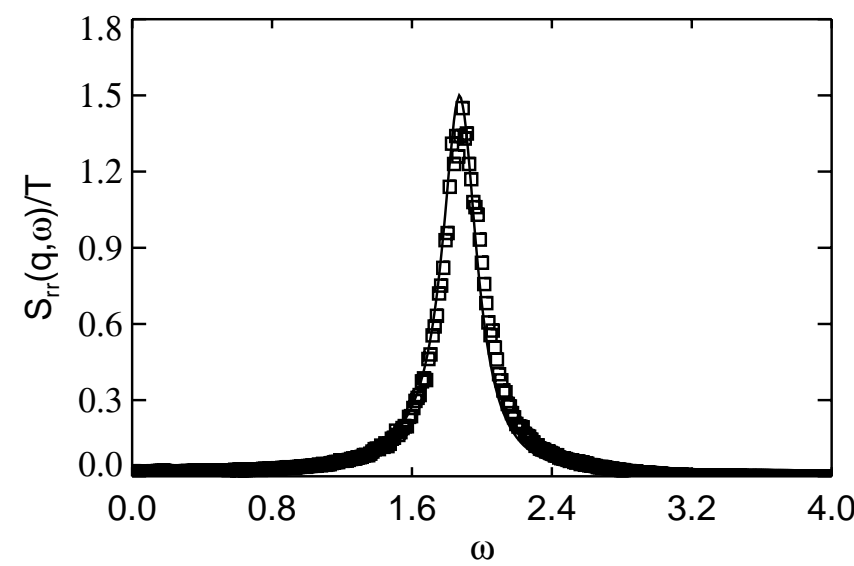

FIG. 1. $\quad r-r$ spectra of the Toda chain at $T=0.25$. Results of molecular dynamics are shown for $q a / \pi=1.0$ (open squares). The full curve is Eq. (7). The linewidth of the zone-boundary phonon is attributed entirely to the diffusion of the phonon phase. of objects supported by the Toda chain, phonons and solitons.

Consider first a phonon $\lambda$ interacting with a gas of objects (solitons or phonons) characterized by a parameter $\alpha$, which in turn specifies the objects' densities $n_{\alpha}$ and velocities $v_{\alpha}$. The phonon experiences a phase shift $\theta(\alpha, \lambda)$ from each object; the current $n_{\alpha} v_{\alpha}$ causes a stochastic frequency shift,

$$
\omega_{\lambda}=\sum_{\alpha} n_{\alpha} v_{\alpha} \theta(\alpha, \lambda),
$$

of the phonon mode $\lambda$. On the average, this process leads to a frequency "drift" $\bar{\omega}_{\lambda}$; in the case of zero-wavelength phonons, we have computed this average drift and found it to be identical with the BA frequency $[13,14]$. In addition, if the densities fluctuate according to

$$
\left\langle\delta n_{\alpha}(t) \delta n_{\alpha^{\prime}}\left(t^{\prime}\right)\right\rangle=\left\langle\left(\delta n_{\alpha}\right)^{2}\right\rangle \delta\left(\alpha-\alpha^{\prime}\right) \delta\left(t-t^{\prime}\right),
$$

the frequency will fluctuate according to

$$
\left\langle\delta \omega_{\lambda}(t) \delta \omega_{\lambda}\left(t^{\prime}\right)\right\rangle=\Gamma_{\lambda} \delta\left(t-t^{\prime}\right),
$$

where

$$
\Gamma_{\lambda}=\sum_{\alpha}\left\langle\left(\delta n_{\alpha}\right)^{2}\right\rangle v_{\alpha}^{2} \theta(\alpha, \lambda)^{2} .
$$

If the statistics of the density fluctuations are Gaussian, the fluctuating phase of the phonon wave function will average to

$$
\left\langle e^{-i \int_{0}^{t} d t^{\prime} \omega_{\lambda}\left(t^{\prime}\right)}\right\rangle=e^{-i \bar{\omega}_{\lambda} t} e^{-\Gamma_{\lambda}|t| / 2} .
$$

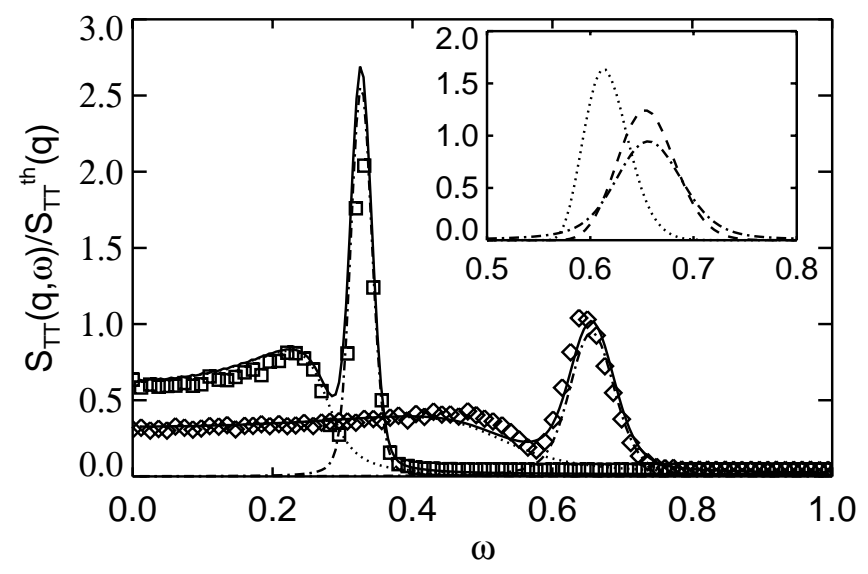

FIG. 2. $T-T$ spectra of the Toda chain at $T=0.25$. Results of molecular dynamics are shown for $q a / \pi=0.1$ (open squares) and $q a / \pi=0.2$ (open diamonds). Dotted lines denote (for both $q$ values) the background (2-phonon) contribution [Eq. (8)]. Dash-dotted lines represent the soliton contribution [Eq. (9)]. Full curves are the sum of the two contributions. The inset demonstrates the quantitative effect of stochastic dynamics on the soliton motion at $q a / \pi=0.2$. The dash-dotted line is a redrawing of the soliton contribution [Eq. (9)] (in agreement with the MD data, cf. body of the figure). The dashed line is the same, except that the diffusion constant has been set equal to zero; i.e., the broadening originates solely in the distribution of soliton velocities. The dotted line has zero diffusion and bare soliton velocities (no "negative fraction"). 
Equations (4) and (6) above are well known in the literature in the context of the phase diffusion model of the laser [17]. In the context of the present Letter, they are supplemented by the identification of the source of randomness (fluctuations in soliton and/or phonon densities, which in turn originate in the randomness of the initial conditions implicit in the canonical ensemble); this allows the calculation of the diffusion rate.

The argument sketched above can be readily applied to solitons, the difference being that (i) phase shifts are substituted by spatial shifts, and (ii) the fluctuating quantity is now the soliton position, rather than the phonon phase. Although the ballistic speed of the solitons is not modified by soliton-soliton or soliton-phonon interactions, the spatial "jumps" associated with each binary collision give rise to a fluctuating shift in the soliton velocity, by analogy with Eq. (2). Consequently, instead of the average drift in phonon frequency there is an average drift in soliton velocity [3]; and instead of the phonon phase diffusion, there is spatial soliton diffusion [1-3]. In both cases, this results, in Fourier space, in broadening Dirac delta functions to Lorentzians.

The above ideas can be applied in order to calculate the observed dynamical correlations by introducing the appropriate densities, velocities, and occupation numbers. Only the basic theoretical outline is sketched here. Details will be reported elsewhere [18].

$r-r$ correlations. - Phonons near the Brillouin zone edge are known [11] to be the best candidates for soliton-phonon phenomenology. In addition, it can be shown that this particular correlation function has no soliton contribution, since the corresponding form factor is vanishingly small. The calculated spectrum is

$$
S_{r r}(q, \omega)=\frac{1}{\pi} \frac{\Gamma_{q} \tilde{\omega}_{q}^{2}}{\left(\omega^{2}-\tilde{\omega}_{q}^{2}\right)^{2}+\Gamma_{q}^{2} \omega} S_{r r}(q) .
$$

In Eq. (7) the static structure factor $S_{r r}(q)$ is given by the product $\omega_{q} \bar{n}_{q}$, where $\omega_{q}$ and $\bar{n}_{q}$ are, respectively, the harmonic frequency and phonon occupation number of the mode $q ; \tilde{\omega}_{q}$ is the thermally renormalized frequency obtained in the BA solution $[13,14]$; the phonon occupation number has been identified as the number of holes in the BA solution. The total phonon phase diffusion constant $\Gamma_{q=\pi / a}=0.120$ is a sum of both soliton (0.094) and phonon (0.026) contributions. We have used known expressions for both phonon-soliton [19] and phononphonon $[11,20,21]$ phase shifts. The calculated spectrum for $q a=\pi$ is shown in Fig. 1. The integrated intensity agrees with the exact (transfer-integral) result [16] — and with MD - to within 1\%. The calculated lineshape is also in agreement with MD. To our knowledge, this is the only case where a phonon lineshape has been accounted for by a nonperturbative mechanism which does not imply decay, rather than a perturbatively computed decay rate.

$T-T$ correlations. - Autocorrelations of the local kinetic energy (i.e., a measure of the local temperature) contain contributions from both 2-phonon (sum and difference processes) as well as a direct soliton part. The 2-phonon contribution is

$$
\begin{aligned}
S_{T T}^{p h}(q, \omega)= & \frac{1}{2 \pi} \int_{-\pi}^{+\pi} d q^{\prime} R\left(q^{\prime}\right) \frac{T}{\tilde{\omega}_{q^{\prime}}} \frac{T}{\tilde{\omega}_{q^{\prime \prime}}} \\
& \times \frac{\Gamma_{q^{\prime}, q^{\prime \prime}}}{\left(\omega-\Omega_{q^{\prime}, q^{\prime \prime}}\right)^{2}+\Gamma_{q^{\prime}, q^{\prime \prime}}^{2}} .
\end{aligned}
$$

In Eq. (8), $R(q)$ is the phonon density of states in $q$ space as modified by the presence of other excitations; $q^{\prime \prime}= \pm(q-q)^{\prime}$, for sum and difference processes, respectively; finally $\Omega_{q^{\prime}, q^{\prime \prime}}$ is the sum or difference of the phonon frequencies involved in the process. The phase diffusion $\Gamma_{q^{\prime}, q^{\prime \prime}}$ in principle includes contributions from both phonon-soliton and phonon-phonon collision processes. Our analysis includes only the phonon-soliton contributions. The 2-phonon (background) contribution to the $T-T$ spectrum (dashed line in Fig. 2) consists of a broad peak at low frequencies and a very broad feature at higher frequencies $(\omega \sim 3.5$, outside the scale of Fig. 2$)$.

The direct soliton contribution is given by

$$
\begin{aligned}
S_{T T}^{s o l}(q, \omega)= & \frac{1}{\pi} \int_{-\infty}^{\infty} d \alpha \bar{n}(\alpha) \\
& \times \frac{D_{\alpha} q^{2}}{\left(\omega-q v_{\alpha}\right)^{2}+\left(D_{\alpha} q^{2}\right)^{2}}\left|f_{T}(q, \alpha)\right|^{2} .
\end{aligned}
$$

In Eq. (9), the integral runs over all soliton parameters, i.e., forward and backward moving solitons; $\bar{n}(\alpha), v_{\alpha}$, and $D_{\alpha}$ are, respectively, the density, velocity, and diffusion constant of solitons with internal parameter $\alpha$; finally, $\quad f_{T}(q, \alpha)=(2 \pi \sinh \alpha / \alpha) / \sinh [(q \pi / 2) / \alpha]-$ $\{\sin (q / 2) \cosh \alpha-[(q / 2) / \alpha] \cos (q / 2) \sinh a\}$ is the static form factor appropriate to local kinetic energy fluctuations. The soliton density is given directly in terms of the BA solution (cf. Ref. [8], where the low-temperature $T^{1 / 3}$ dependence of the total soliton density is derived). The soliton velocity follows from a literal interpretation of the BA quasimomenta $h(k)$ and quasienergies $\boldsymbol{\epsilon}(k)$ above the Fermi level as soliton momenta $[8,21]$ and energies, respectively; the resulting slope $\partial \epsilon / \partial h$ defines the soliton velocity at finite temperatures [13]. This thermally renormalized velocity is systematically higher than the bare soliton velocity. In effect, the BA solution, by incorporating all phase-shift interactions exactly, has built-in effects such as the velocity drift for solitons, or the frequency drift for phonons (cf. above). Finally, we note that the diffusion constant calculated according to Eq. (5) contains contributions from both soliton-phonon and soliton-soliton parts.

The direct soliton contribution to the spectrum [Eq. (9), dotted line in Fig. 2] can be unambiguously identified with the main peak observed in MD. The total calculated spectrum [sum of Eqs. (8) and (9), solid line in Fig. 2] 
is in reasonable agreement with MD. The integrated intensity is $7 \%$ higher than the exact ( $q$-independent) theoretical value $S_{T T}^{\text {th }}(q)=T^{2} / 2$ [16] for $q a / \pi=0.1$; it coincides with the exact value for $q a / \pi=0.2$; for higher values of $q$, agreement becomes worse, probably reflecting the limitations of soliton-phonon phenomenology.

A number of comments are in order here.

(i) The exact correspondence of the position of the predicted soliton peak with the MD result can be obtained only with the fully renormalized soliton velocities of the BA. Using the bare velocities in Eq. (9) results in soliton peak positions shifted to lower frequencies, as well as in systematic errors in the $q$ dependence and intensity of the peak (cf. inset of Fig. 2). Thus, although the effect of thermal renormalization on the soliton velocity is small (approximately $5 \%$ at $T=0.25$ ), the evidence provided for it by the present work is compelling. This in turn implies that BA quasiparticles, although derived strictly within the context of equilibrium statistical mechanics, are legitimate dynamical entities at finite temperatures. Furthermore, to the extent that the BA solution contains the velocity drift effect, this Letter provides indirect evidence for the negative friction exerted by the phonon gas on the soliton, a suggestion first made in the context of the sine-Gordon model [3].

(ii) A point we would like to emphasize is the significance of identifying a purely solitonic feature in the spectrum. In view of the fact that various authors have stressed the generic character of (nontopological) solitonlike objects in one-dimensional chains-even if they do not possess the exact soliton property-our findings imply that it is possible to investigate whether other, less "pathological" models exhibit similar solitonlike signatures in the spectra of dynamical correlations of the local kinetic energy. Preliminary results for the Lennard-Jones chain confirm this expectation.

(iii) A final comment concerns the magnitude of the diffusion constants. The average soliton diffusion constant at $T=0.25$ is, in dimensionless units, equal to 0.09 . The linewidth associated with it is quite small; it accounts for approximately $25 \%$ of the total linewidth; the rest originates in the natural distribution of soliton velocities (cf. inset of Fig. 2). Thus, although observable, the effect of nondissipative diffusion on soliton spectra is small. In contrast, the diffusion of the phonon phase leads to a much more pronounced broadening of phonon features (cf. Fig. 1).

In conclusion, our parameter-free analysis of the finitetemperature spectral properties of the Toda lattice has identified characteristic solitonic features. Our results strongly suggest that the BA quasiparticles can be used to describe the unusual stochastic dynamics (negative friction, nondissipative diffusion) of soliton-bearing systems.

Part of this work has been supported by the GreekFrench bilateral cooperation program Plato.

[1] Y. Wada and J.R. Schrieffer, Phys. Rev. B 18, 3897 (1978).

[2] N. Theodorakopoulos, Z. Phys. B 33, 385 (1979).

[3] K. Fesser, Z. Phys. B 39, 47 (1980).

[4] M. Ogata and Y. Wada, J. Phys. Soc. Jpn. 55, 1252 (1986).

[5] F. Marchesoni and C. R. Willis, Europhys. Lett. 12, 491 (1990).

[6] M. Toda, Theory of Nonlinear Lattices (Springer-Verlag, Berlin, 1981).

[7] V. Muto, A. C. Scott, and P. L. Christiansen, Phys. Lett. A 136, 33 (1989); Physica (Amsterdam) 44D, 75 (1990).

[8] N. Theodorakopoulos and N.C. Bacalis, Phys. Rev. B 46, 10706 (1992); an approximate calculation of the soliton density has been performed by F. Marchesoni and C. Lucheroni [Phys. Rev. B 44, 5303 (1991)].

[9] H. Flashka, Phys. Rev. B 9, 1924 (1974).

[10] B. Sutherland, Rocky Mt. J. Math. 8, 413 (1978).

[11] N. Theodorakopoulos, Phys. Rev. Lett. 53, 871 (1984).

[12] M. Opper, Phys. Lett. 112A, 201 (1985).

[13] P. Grüner-Bauer and F. G. Mertens, Z. Phys. B 70, 435 (1988).

[14] M. Fowler and N.-C. Yu, J. Phys. A 22, 3095 (1989).

[15] J. Krumhansl and J.R. Schrieffer, Phys. Rev. B 11, 3535 (1975).

[16] T. Schneider, in Solitons, edited by S.E. Trullinger, V.E. Zakharov, and V. I. Pokrovsky (Elsevier, New York, 1986), p. 389; T. Schneider and E. Stoll (unpublished). These authors tentatively identify the broad feature in the $T-T$ spectrum with second sound and the main peak with a "bound-state-like resonance." Although the position given for the second-sound peak would be roughly consistent with observation, our calculation for the total spectrum is internally consistent without invoking second-sound contributions.

[17] For example, G. S. Agarwal, Phys. Rev. Lett. 37, 1383 (1976).

[18] N. Theodorakopoulos (to be published).

[19] N. Theodorakopoulos and F. G. Mertens, Phys. Rev. B 28, 3512 (1976).

[20] N. Theodorakopoulos (unpublished).

[21] H. Takayama and M. Ishikawa, Prog. Theor. Phys. 77, 820 (1986). 